\title{
Platelet and haemoglobin levels in Patients on Isosorbide Mononitrate and/or Cilostazol with Lacunar Ischaemic Stroke: Data from the LACI-1 trial
}

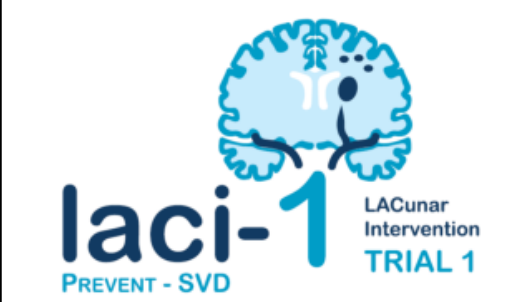

Jason P Appleton ${ }^{1}$, Katie Flaherty ${ }^{1}$, Gordon W Blair ${ }^{2}$, Zhe Kang Law ${ }^{1}$, Fergus Doubal ${ }^{2}$, Nikola Sprigg ${ }^{1}$, Philip M Bath ${ }^{1}$, Joanna M Wardlaw ${ }^{2}$

1. Stroke Trials Unit, Division of Clinical Neuroscience,

THE UNIVERSITY of EDINBURGH \section{University of Nottingham, Nottingham, UK \\ 2. Centre for Clinical Brain Sciences, University of Edinburgh, Edinburgh UK}

Alzheimer's

Society United Against
Dementia

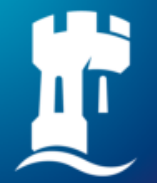

University of Nottingham

\section{BACKGROUND}

Cilostazol and isosorbide mononitrate (ISMN) may benefit cerebral small vessel disease and lacunar ischaemic stroke.

- Both drugs may also influence platelet and haemoglobin levels.

- We assessed their effects in the lacunar intervention trial-1 (LACI-1).

\section{RESULTS}

We recruited 57 patients in total; those allocated cilostazol alone were older but otherwise the groups were well-balanced at baseline.

Table: Haematology and platelet function results at week 8

\begin{tabular}{|c|c|c|c|}
\hline & $\begin{array}{c}\text { Cilostazol vs. } \\
\text { none }\end{array}$ & $\begin{array}{l}\text { ISMN vS. } \\
\text { none }\end{array}$ & $\begin{array}{c}\text { Both vs. } 1 \text { or } \\
\text { other }\end{array}$ \\
\hline FBC & MD (95\% CI) & MD (95\% CI) & MD (95\% CI) \\
\hline $\mathrm{Hb}$ & $\begin{array}{c}-3.72 \\
(-8.39,0.95) \\
\end{array}$ & $\begin{array}{c}-1.03 \\
(-6.92,4.87) \\
\end{array}$ & $\begin{array}{c}-2.35 \\
(-6.82,2.11) \\
\end{array}$ \\
\hline Plt & $\begin{array}{c}35.73 \\
(2.81,68.66) \\
\end{array}$ & $\begin{array}{c}3.92 \\
(-32.35,40.19)\end{array}$ & $\begin{array}{c}-7.43 \\
(-36.76,21.89)\end{array}$ \\
\hline \multicolumn{4}{|c|}{ Platelet function } \\
\hline Ref. & $\begin{array}{c}-2.90 \\
(-15.09,9.30)\end{array}$ & $\begin{array}{c}-8.23 \\
(-20.68,4.22)\end{array}$ & $\begin{array}{c}7.51 \\
(-4.01,19.02)\end{array}$ \\
\hline$A A$ & $\begin{array}{c}-83.57 \\
(-256.5,89.37)\end{array}$ & $\begin{array}{c}-14.13 \\
(-192.5,164.24)\end{array}$ & $\begin{array}{c}109.73 \\
(-54.01,273.48)\end{array}$ \\
\hline ADP & $\begin{array}{c}-1.33 \\
(-77.64,74.98)\end{array}$ & $\begin{array}{c}-0.50 \\
(-80.14,79.13)\end{array}$ & $\begin{array}{c}11.92 \\
(-60.23,84.07)\end{array}$ \\
\hline
\end{tabular}

Bold indicates $p<0.05 . A A$ : arachidonic acid (aspirin test); ADP: adenosine diphosphate (clopidogrel test); $\mathrm{CI}$ : confidence interval; FBC: full blood count; $\mathrm{Hb}$ : haemoglobin; ISMN: isosorbide mononitrate; MD: mean difference; PIt: platelet; Ref:

\section{METHODS}

LACI-1 randomised patients with lacunar ischaemic stroke to immediate ISMN, cilostazol, both drugs for 9 weeks immediate start or both drugs delayed start at week 4 . Full blood counts were taken at baseline, and weeks 3 and 8:

\section{- Haemoglobin $(\mathrm{Hb}, \mathrm{g} / \mathrm{L})$ \\ - Platelets (PIt, x10\%/L)}

- Platelet function was assessed with remote measurement of surface expression of $\mathrm{P}$ selectin (CD62P) using kits sensitive to aspirin (arachidonic acid) or clopidogrel (adenosine diphosphate) at the same timepoints.

- Differences in haemoglobin and platelet levels and platelet function were assessed by multiple linear regression, adjusted for baseline value and key covariates.

Data are mean (standard deviation, SD) or mean difference (MD) with 95\% confidence intervals (CI).

\begin{tabular}{|llccc|}
\hline \multicolumn{4}{|c}{ RESULTS } \\
\hline - Week 8: & n & Hb & Plt \\
- & Cilostazol & 13 & $131(10)$ & $290(61)$ \\
- & ISMN & 15 & $147(12)$ & $239(46)$ \\
- & Both immediate 14 & $137(15)$ & $284(58)$ \\
- & Both delayed & 14 & $136(14)$ & $258(56)$
\end{tabular}

At week 8:

- Hb did not differ between the treatment groups.

- Plt levels were slightly higher in those who received cilostazol vs. no cilostazol.

- No differences were noted in platelet function between treatment groups.

\section{CONCLUSIONS}

- Cilostazol and isosorbide mononitrate have no clinically concerning effects on haemoglobin and platelet levels and function in the short to medium term.

- Further assessment of the safety and efficacy of these medications following lacunar ischaemic stroke is warranted. 